\title{
Vestibular Schwannoma Drug Development: Current State-of-the Art
}

\author{
Craig Miller $\cdot$ Holger Sudhoff $\cdot$ Abraham Jacob
}

Published online: 10 September 2014

(C) Springer Science+Business Media New York 2014

\begin{abstract}
Vestibular schwannomas (VS) can cause significant patient morbidity. Currently, surgery and stereotactic radiation therapy are available treatment options. Recent breakthroughs in research have discovered key cell surface receptors and intracellular signaling pathways that drive vestibular schwannoma tumorigenesis, proliferation, and survival. A number of promising inhibitors targeting these signaling molecules have also now shown efficacy in preclinical VS cell culture models and animal experiments, with some recently entering human clinical trials. In this review, we summarize ErbB receptor signaling, PDGF receptors, MAP kinase signaling, AKT, p21-activated kinase signaling, mTOR, and VEGF signaling in the context of vestibular schwannoma drug development and medical treatment.
\end{abstract}

Keywords Vestibular schwannomas - Neurofibromatosis type II $\cdot$ NF2 $\cdot$ AKT $\cdot$ mTOR $\cdot$ PAK

C. Miller · A. Jacob

Department of Surgery/Division of Otolaryngology, Otology, Neurotology, \& Cranial Base Surgery, The University of Arizona Ear Institute, 1515 North Campbell Avenue, P. O. Box 245024, Tucson, AZ 85724, USA

e-mail: cmiller@surgery.arizona.edu

A. Jacob

e-mail: ajacob@surgery.arizona.edu

H. Sudhoff $(\bowtie)$

Department of Otolaryngology, Head and Neck Surgery, Bielefeld Academic Teaching Hospital, Affiliated to Bielefeld and Münster University, Teutoburger Str. 50, 33604 Bielefeld, Germany

e-mail: holger.sudhoff@rub.de

\section{Introduction}

Vestibular schwannomas (VS), benign tumors originating from the vestibulocochlear nerve, typically present with hearing loss, tinnitus, and balance dysfunction. Rarely, however, if untreated, these neoplasms can cause significant patient compromise-facial paralysis, brainstem compression, and death. Those with VS typically choose between surgery and stereotactic radiation therapy for treatment. Unfortunately, no medical therapies are presently U.S. Food \& Drug Administration or European Medicines Agency (EMA) approved, representing an urgent and unmet clinical need. Recent breakthroughs in research have discovered key signaling pathways (Fig. 1) that drive vestibular schwannoma proliferation/survival. A number of inhibitors targeting these molecules have shown efficacy in preclinical VS models, with some recently entering human clinical trials. In this review, we discuss the current state of vestibular schwannoma drug development efforts worldwide.

\section{NF2 and Merlin}

NF2 is an autosomal-dominant disease caused by the bi-allelic loss of the NF2 gene on chromosome 22. Bilateral VS are pathognomonic for this disorder, characterized by loss of the $N F 2$ gene that codes for the tumor suppressor protein merlin (moesin, ezrin, and radixin-like protein) $[1,2]$. Though $50 \%$ of individuals with NF2 have an affected parent with the disease, the remaining $50 \%$ have de novo gene mutations [3]. While patients with germ-line mutations in NF2 develop bilateral VS, unilateral, sporadic tumors are far more common, and NF2 gene mutations are present in $100 \%$ of VS tumors.

Tumorigenesis occurs due to molecular abnormalities that deregulate cell growth and differentiation [4]. 


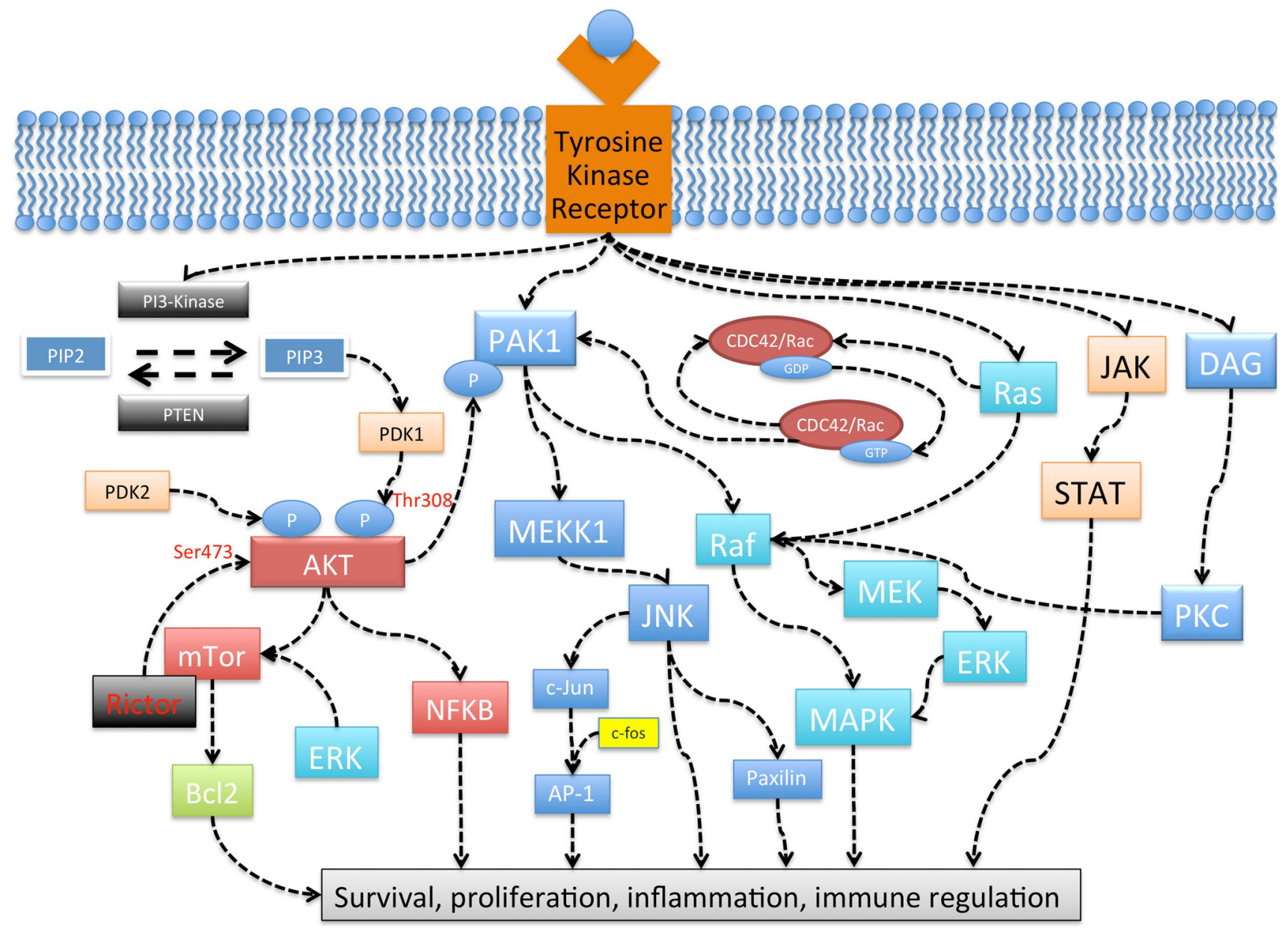

Fig. 1 Multiple growth promoting intracellular pathways are activated by number of tyrosine kinase receptors, generically depicted in this image. Merlin (not pictured) when present acts as a master suppressor for many of these pathways, thus controlling growth and

Differentiated Schwann cells become quiescent upon sensing cell contact, and it is the NF2 gene product, merlin, that regulates this contact-dependent inhibition of proliferation [5-7]. Like other members of the band 4.1superfamily, merlin tethers proteins to the actin cytoskeleton or sequesters them to specific intracellular compartments. Most believe that merlin functions as a tumor suppressor [8, 9] by cycling between a closed, growth-suppressive conformation and an open, growth permissive state induced by phosphorylation at serine-518 [10-12]. Others, however, hypothesize that merlin exists in multiple context-specific conformations [13]. At the molecular level, extracellular cues regulate merlin's phosphorylation status and, thereby, its activity. For example, integrin and receptor tyrosine kinase signaling activate p21-activated kinase (Pak), which has been shown to phosphorylate merlin on Ser518; conversely, cadherin signaling normally inhibits Pak and leads to the accumulation of the unphosphorylated, active (growth- cell proliferation. When deficient, as in NF2, many of these pathways are disregulated and aberrant cell proliferation and survival occurs leading to the tumors seen in Neurofibromatosis type II

suppressive) merlin [14]. Merlin may regulate cell proliferation [15], at least in part, by controlling availability of membrane receptors such as EGFR (epidermal growth factor receptor), erbB2, and PDGFR (platelet-derived growth factor receptor) [16] through its interaction with adaptor molecules such as NHERF1/EBP50 (Na-H exchange regulatory factor 1; ERM-binding protein of $50 \mathrm{kDa})$ [17-19].

\section{Current Clinical Trials}

Drug development for NF2 is not a new concept as several pathway inhibitors are under investigation at this time.

The ErbB Family of Tyrosine Kinase Receptors

The ErbB membrane tyrosine kinase receptors regulate cell proliferation, differentiation, motility, and apoptosis 
[20]. Hanson et al. [21] discovered that neuregulin and its receptors erbB2 and erbB3 were expressed in human vestibular schwannomas. They and others hypothesized that neuregulin, secreted by tumor cells, functioned as an autocrine growth factor that promoted survival of schwannoma cells despite their lack of association with surrounding nerves. Lallemand et al. [22] showed that the NF2 protein modified the abundance and localization of ErbB2, ErbB3, insulin-like growth factor 1 receptor (IGF1R), and platelet-derived growth factor-b (PDGFRb) receptors at the plasma membrane. Using NF2 knockout mice, they showed that varying insulin (IGF1R ligand) concentrations had no effect on schwannoma phenotype, but lowering levels of the ErbB ligand, heregulin-b1, restored contact inhibition and replicative senescence. Preclinical studies have validated ErbB family receptors as potential targets for drug development. Ammoun and colleagues used in vitro phosphoRTK profiling arrays to confirm overexpression and activation of EGFR family receptors in human VS samples. They also evaluated the small-molecule EGFR/ ErbB2 kinase inhibitor lapatinib (GlaxoSmithKline) in vitro, finding that this drug inhibited ErbB2 phosphorylation, downstream ERK1/2 and AKT activation, and VS cell proliferation. Clark and coworkers also independently validated the use of ErbB family receptor inhibitors in vivo [23].

Several small clinical studies have investigated the efficacy of ErbB family receptor blockade for treatment of VS. Plotkin and colleagues treated eleven NF2 patients with erlotinib $150 \mathrm{mg} /$ day [24]. While erlotinib treatment did not result in radiographic or hearing responses in NF2 patients with progressive disease, a subset of patients experienced prolonged stable disease. These investigators concluded that time-to-progression might be more appropriate than radiographic or hearing responses for anti-EGFR agents in NF2-associated VS. Karajannis and coworkers recently reported on results of a phase II trial evaluating the effectiveness of lapatinib therapy. They found decreased tumor volumes and hearing improvements rates of 23.5 and $30.8 \%$, respectively. Three of 17 patients had no disease progression, and adverse events were both mild and rare [25]. The website "clinicaltrials.gov" presently lists two clinical trials for the EGFR/ErbB2 kinase inhibitor lapatinib in VS patients. Trials evaluating response to lapatinib treatment in NF2 patients are currently active or recruiting at New York University as a phase II study for NF2 patients, and Sidney Kimmel Comprehensive Cancer Center in Baltimore, MD, as an exploration and estimation of VS intratumoral concentration and activity of lapatinib in vivo. Results from these studies have not yet been reported.
Targeting Angiogenesis: The Vascular Endothelial Growth Factor

Vascular endothelial growth factor (VEGF), a known mitogen, is a heparin-binding glycoprotein that is secreted as a $45 \mathrm{kD}$ homodimer [26]. This molecule promotes survival of various cell types, including Schwann cells [27] and correlates with vascularity and growth rate of several solid tumors [28]. VEGF promotes survival through proangiogenesis and anti-apoptosis effects [29, 30]. CayeThomasen et al. reported increased VEGF and VEGF-R1 expression in human vestibular schwannomas; [31] their work generated significant clinical interest in use of VEGF inhibitors for VS treatment.

Bevacizumab, a monoclonal antibody against VEGF, has demonstrated substantial reduction of tumor volume as well as improved hearing outcomes in some patients with VS [32-35]. Bevacizumab does not have a direct antiproliferative effect on tumor cells, but it does reduce tumor volume by increasing vascular permeability and decreasing angiogenesis. Unfortunately, as reported by Mautner and colleagues, discontinuing the drug results in re-expansion of VS tumors [36]. The website clinicaltrials.gov reports five studies related to Bevacizumab and schwannoma. Two trials are currently recruiting patients. These are a Phase I trial investigating the safety of intraarterial cerebral infusion of bevacizumab for VS being conducted at Weill Medical College of Cornell University, and a phase II study to determine the hearing response rate at 24 weeks after treatment with bevacizumab for symptomatic VS in children and young adults with NF2 at the University of Alabama Birmingham. One phase II study conducted by the National Cancer Institute investigated bevacizumab in children and adults with NF2 and symptomatic VS. This study is completed, but results have not yet been reported.

PTC299 is a small-molecule that demonstrated reduction of tumor volume by inhibiting VEGF and other angiogenic cytokine production at the post-transcriptional level. It binds the $5^{\prime}$ - and $3^{\prime}$-untranslated regions of VEGF messenger RNA, preventing protein translation. This drug has been shown to induce cycle arrest at the G1/S phase [80]. A human clinical trial for PTC299 in patients with NF2-associated tumors was initiated by PTC Therapeutics in 2009 and is still listed on clinicaltrials.gov; however, the study is currently suspended.

Endostatin, a $20 \mathrm{kDa}$ inhibitor of endothelial cell proliferation and organization into new blood vessels, has been shown to suppress tumor growth and is being investigated as a possible treatment for NF2-related tumors. Produced by proteolytic cleavage of collagen XVIII, endostatin is thought to down-regulate several signaling cascades including TNF-alpha/JNK, FGF, integrin, NFkappaB, and ephrin [37]. According to clinicaltrials.gov, one trial, 
initiated in March 2014 and sponsored by Beijing Tiantan Hospital, is currently recruiting patients to evaluate recombinant human endostatin injection for patients with NF2 and NF2-related tumors by continuous intravenous pumping. The endpoints for this study are tumor volume, hearing improvement, and various quality of life measures. No results have been reported to date.

\section{mTOR Signaling}

The literature suggests that normally functioning merlin protein regulates mTOR (mammalian target of rapamycin) activity, independent of the AKT or MAPK pathways. Lopez-Lago found that loss of merlin in NF2 deficient cell lines was associated with activation of mTORC1. They demonstrated that rapamycin, an inhibitor of mTORC1, inhibited cell growth in the same cell lines, while overexpression of merlin rendered the cells partially resistant to rapamycin treatment. Depletion of merlin restored rapamycin sensitivity in merlin-positive lines [38]. A number of other studies have suggested an up-regulation of mTORC signaling in merlin-deficient patients and cell lines [39].

AKT activation requires two phosphorylation eventsPDK1 mediated Thr308 phosphorylation and PDK2 mediated Ser473 phosphorylation. Many believe that mTOR, when bound to its adaptor protein Rictor, may function as a PDK2 candidate for schwannomas [40]. Theoretically, inhibiting mTOR could suppress tumor growth by both preventing AKT pathway activation and inhibiting mTOR specific, non-AKT signals.

Everolimus (RAD001; Novartis) is an mTOR inhibitor used to treat advanced renal cell carcinoma and subependymal giant cell astrocytoma. A recently conducted Phase II study of everolimus in NF2 patients looked at volumetric and audiological response in schwannomas. The study, conducted at New York University between January and June 2012, identified no objective response in any of the nine patients enrolled [41]. Other clinical trials are ongoing and actively recruiting. Clinicaltrials.gov currently lists five studies looking to evaluate the safety and efficacy of RAD001 in patients with NF2-associated vestibular schwannomas. Two studies are actively recruiting patients, two are not recruiting patients any longer but are currently ongoing, and one is completed but no results have been published. The studies that are currently recruiting are taking place at the House Ear Institute as a Single Arm Phase II Trial of RAD001 as monotherapy in the Treatment of Neurofibromatosis Type 2-related Vestibular Schwannoma and New York University School of Medicine exploring the activity of RAD001 in NF2-associated VS and meningiomas. The two active, but no longer recruiting, studies are being conducted at Assistance Publique-Hôpitaux de Paris as a single arm Phase II Trial of
RAD001 as monotherapy for treatment of Neurofibromatosis Type 2-related vestibular schwannoma and New York University School of Medicine in collaboration with The Children's Tumor Foundation as a phase II study of everolimus in children and adults with NF2.

\section{Future Opportunities for Drug Development}

\section{AKT Signaling}

AKT (Protein Kinase B), a master kinase regulating cell growth and survival, has been of primary interest to the NF2 community [42]. This signaling molecule targets downstream effectors such as GSK3 $\beta$ (Glycogen synthase kinase 3), BAD (Bcl-2-associated death promoter), TSC1/ TSC2-mTOR (Tuberous sclerosis proteins; mammalian target of rapamycin), caspase-9, and FOXO3a (forkhead box proteins). Deregulated AKT signaling has been shown to induce malignant transformation in various cell types [43]. AKT is activated by PI3-kinase, a membrane-associated lipid kinase that is activated downstream of most receptor tyrosine kinases. PI3-kinase catalyzes the conversion of PIP2 to PIP [3, 43] which recruits AKT from the cytoplasm to the plasma membrane via pleckstrin homology $(\mathrm{PH})$ domain interactions $[44,45]$. AKT is then activated by phosphoinositide-dependent protein kinase 1 (PDK1), at thr308 [46]. A second phosphorylation at serine-473 is required for maximal AKT activation [47].

The PI3-kinase/AKT pathway has been shown as activated in human vestibular schwannomas. Using microarrays, Western blots and immunohistochemistry, Ohio State University investigators demonstrated a modest increase in total AKT protein for VS compared with normal vestibular nerves, but phosphorylated AKT is dramatically increased. They concluded that post-translational AKT phosphorylation was the primary mechanism whereby AKT is activated in human VS [48]. Immunohistochemistry studies by other investigators compared 16 schwannomas (sporadic and NF2) to traumatic neuromas and found that schwannomas consistently demonstrated increased expression of p-AKT [49].

Because the PI3K/AKT pathway is the junction for numerous upstream survival, differentiation, cell cycle, stress, and apoptosis signals, several groups have postulated that AKT pathway inhibition should suppress VS growth. To test this hypothesis, work done at The Ohio State University employed a pharmacology-based strategy using two novel small-molecule inhibitors of the AKT pathway that diverged significantly in their proposed mechanisms of action. AR12 (OSU-03012; ARNO Therapeutics, Parsippany, NJ), derived from the cyclooxygenase2 (COX-2) inhibitor Celebrex ${ }^{\mathrm{TM}}$, inhibits tumor growth 
due, at least in part, to inhibition of phosphoinositidedependent kinase-1 (PDK1) [50-55]. AR42 (OSUHDAC42; ARNO Therapeutics, Parsippany, NJ), a novel histone deacetylase inhibitor (HDACi), inhibits AKT downstream from PI3K and PDK1 by activating protein phosphatase 1-mediated AKT dephosphorylation [56]. Using in vitro and in vivo model systems, they demonstrated that both AR12 and AR42 were able to suppress schwannoma growth in vitro/in vivo, inhibit AKT activation, slow cell cycle arrest, and induce apoptosis [57-59]. AR12 and AR42 penetrated the blood-brain barrier, and exhibited minimal pharmacotoxicity. Both these drugs are currently in Phase I clinical trials for human cancers and have demonstrated excellent safety profiles; AR42 has been given to several patients with NF2.

Several other PI3-kinase and AKT inhibitors are in various stages of development against non-schwannoma tumors. Perifosine (KRX-0401), an alkyl phospholipid that inhibits both PI3-kinase and AKT, entered Phase III clinical trials against colorectal cancer and multiple myeloma as an orphan drug; unfortunately, these trials discontinued due to lack of efficacy. The company, Æterna Zentaris Inc., reported no safety concerns [60]. Miltefosine, (structurally similar to perifosine), is an AKT inhibitor used as an antiprotozoal drug as well as treatment for HIV. There are no current studies being conducted for use as a chemotherapeutic agent. PI3-kinase inhibitors such as CAL101 and PX-866 are in late stage clinical trials for hematologic malignancies and late stage solid tumors, respectively. BEZ235 (PI3-kinase/mTOR dual inhibitor), SF1126, INK1117, IPI-145, GDC-0941, BKM120, XL147, XL765, Palomid 529, GSK615, ZSTK474, and PWT33597 (dual PI3-kinase/mTOR) are in earlier stages of development. No vestibular schwannoma related clinical studies have been published to date for any AKT inhibitor.

\section{PAK Signaling}

The six known Group 1 and Group 2 P21-activated kinases (PAKs) phosphorylate serine/threonine residues on various intracellular targets [61]. PAKs are immediate effectors of the Rho family GTPases Rac and Cdc42, which mediate cytoskeleton reorganization and transcriptional activation [62]. In 2002, Xiao and colleagues discovered that PAK could phosphorylate merlin at serine-518, eliminating its tumor suppressor function [63]. Conversely, researchers also discovered that merlin inhibited PAK by means of a negative-feedback loop [64]. It was observed that NF2deficient tumors have morphology similar to cells overexpressing the GTPase Rac [65-67]. Following this observation, Kaempchen et al. hypothesized that enhanced Rac activity is present in merlin-deficient tumors and confirmed that this increase contributes directly to schwannoma-genesis [68]. They and others demonstrated that Rac and PAK signaling co-localize to the membrane, where together Rac/PAK controls cellular processes such as spreading, formation of ruffles, and adhesion to the cellular matrix [68-70]. The notion that wild-type merlin inhibits the Rac-binding domain of PAK1 is supported, by the finding that reintroduction of functioning merlin protein in cells with NF2 loss results in increased PAK activity [71]. PAK knockdown has been shown to counter tumorigenesis in merlin-deficient cell lines [69].

Several compounds that inhibit PAK signaling have emerged as viable leads for further drug development. One is IPA-3. Using primary screens, Bokoch and colleagues identified 342 candidate PAK inhibitors [72] that possessed the ability to inhibit PAK activity allosterically. After excluding ATP-competitive inhibitors, they found 32 lead compounds, the most notable of these being $2,2^{\prime}$-dihydroxy-1,1'-dinaphthyldisulfide (coined IPA-3). IPA-3 has a high degree of specificity for group I PAKs, prevents PAK autophosphorylation, and sequesters it from Rac- and Cdc42-mediated activation. Flaiz and colleagues showed that IPA-3 was effective at reducing Rac activity, cell spreading, and cell adhesion in primary human schwannoma cells [73]. Washout experiments following IPA-3 administration restored the normal schwannoma phenotype, indicating that the effects of IPA-3 were reversible. Porchia et al. demonstrated that the PDK1 inhibitor AR12 (aka OSU-0312) also functions as a competitive inhibitor of PAK activity [53]. Structural modification of AR12 to make an even more potent PAK inhibitor is currently underway. Currently, the group at the University of Arizona is evaluating the efficacy and mechanism(s) of action for two novel derivatives of AR12 that appear to be more potent PAK inhibitors than their parent compound.

Hsp90

Molecular chaperones are proteins dedicated to protein folding and refolding [74]. Heat shocking proteins are a family of these chaperones responsible for directing the folding of much of the proteome, resulting in the formation of proteins and protein complexes responsible for cell metabolism. Heat shock protein 90 (HSP90), is a ubiquitous molecule that when absent, results in proteasomal degradation of its "client" proteins [75]. Angelo and colleagues were able to inhibit heat shock protein in a schwannoma cell line. This resulted in cessation of cell growth, opening the door for more investigations into this avenue for therapy. Recent work by Tanaka and colleagues sought to better understand the efficacy of HSP90 inhibition as a treatment strategy to suppress multiple growth promoting signal pathways. Using a novel small-molecule inhibitor compound of HSP90, NXD30001 (pochoxime A), 
they were able to show in vitro depletion of multiple signaling molecules implicated in NF2 [76]. Additionally, they were able to show reduced growth of NF2-deficient tumors and associated pathways in vivo. There are no current clinical trials using HSP90 inhibitors, but promising preclinical results will surely open doors to the opportunity.

\section{Natural Compounds}

Yet another promising area for drug development is the identification of natural compounds with antitumor activity and screening of these compounds using bioassay techniques. A number of current chemotherapeutic agents for a wide range of cancers are based on natural compounds [77]. Recent results published by Spear and colleagues investigated two natural compounds, Cucurbitacin $\mathrm{D}$ and goyazensolide, and evaluated the cell cycle and antitumor effects of both compounds in NF2-deficient meningioma and schwannoma cells [78]. They were able to show that both compounds suppressed the growth of cells in vitro, induced G2 cell cycle arrest, and affected the expression of multiple signaling molecules such as Cyclins E, A, and B and NFאB. Further work is needed, but the promising results of natural compounds as anticancer regimens in the past make this an exciting area of investigation.

\section{High-Throughput Drug Screens}

Basic science and clinical research efforts to date have been based on decades of hypothesis-driven drug development strategies. Empirical techniques using highthroughput screening (HTS), chemical synthesis, RNA interference, and bioinformatics have revolutionized drug development efforts worldwide for cancer; however, these non-traditional methodologies for lead identification in drug development have rarely been used for benign tumors. Recently, Petrilli and colleagues used a high-throughput screen (with NF2 null mouse Schwann cells) of the Library of Pharmacologically Active Compounds to discover that AGK2, a SIRT2 (sirtuin 2) inhibitor, was a candidate compound for further drug development in schwannomas [79]. SIRT2, one of seven mammalian sirtuins that are NAD+-dependent protein deacetylases, was identified as a target using the empirical approach; then, this group showed that merlin-mutant mouse Schwann cells (MSC) have higher expression levels of SIRT2 and lower levels of overall lysine acetylation than wild-type control MSC. Others have also adopted this empirical strategy for VS drug development; therefore, perhaps a wave of new targets for vestibular schwannoma clinical trials is soon to come $[80,81 \bullet, 82 \bullet, 83 \bullet \bullet, 84 \bullet, 85 \bullet \bullet, 86 \bullet]$.

\section{Conclusion}

The application of small-molecule inhibitors of deregulated pathways is the current trajectory in many disciplines of cancer therapy, and otology and neurotology is no exception. It is a truly exciting time in the specialty as continued research into the critical signaling pathways driving schwannoma proliferation/survival has generated a viable pipeline of candidate drugs targeting these tumors.

\section{Compliance with Ethics Guidelines}

Conflict of Interest Craig Miller, Holger Sudhoff, and Abraham Jacob declare that they have no conflict of interest.

Human and Animal Rights and Informed Consent This article does not contain any studies with human or animal subjects performed by any of the authors.

\section{References}

Recently published papers of particular interest have been highlighted as:

- Of importance

-• Of major importance

1. Rouleau GA, Merel P, Lutchman M, et al. Alteration in a new gene encoding a putative membrane-organizing protein causes neuro-fibromatosis type 2. Nature. 1993;363:515-21.

2. Trofatter JA, MacCollin MM, Rutter JL, et al. A novel moesin-, ezrin-, radixin-like gene is a candidate for the neurofibromatosis 2 tumor suppressor. Cell. 1993;72:791-800.

3. Evans DG. Neurofibromatosis type 2 (NF2): a clinical and molecular review. Orphanet J Rare Dis. 2009;4:16.

4. Hanahan D, Weinberg RA. The hallmarks of cancer. Cell. 2000;100:57-70.

5. Levine EM, Becker Y, Boone CW, Eagle H. Contact inhibition, macromolecular synthesis, and polyribosomes in cultured human diploid fibroblasts. Proc Natl Acad Sci USA. 1965;53:350-6.

6. Levine BB. Studies on delayed hypersensitivity. I. Inferences on the comparative binding affinities of antibodies mediating delayed and immediate hypersensitivity reactions in the guinea pig. J Exp Med. 1965;121:873-88.

7. Eagle H, Levine EM, Boone CW. Cellular growth, contact inhibition, and macromolecular synthesis. Science. 1965;148:665.

8. Bretscher A, Chambers D, Nguyen R, Reczek D. ERM-Merlin and EBP50 protein families in plasma membrane organization and function. Annu Rev Cell Dev Biol. 2000;16:113-43.

9. Bretscher A, Edwards K, Fehon RG. ERM proteins and merlin: integrators at the cell cortex. Nat Rev Mol Cell Biol. 2002; 3:586-99.

10. Shaw RJ, McClatchey AI, Jacks T. Localization and functional domains of the neurofibromatosis type II tumor suppressor, merlin. Cell Growth Differ. 1998;9:287-96.

11. Shaw RJ, McClatchey AI, Jacks T. Regulation of the neurofibromatosis type 2 tumor suppressor protein, merlin, by adhesion and growth arrest stimuli. J Biol Chem. 1998;273:7757-64.

12. Shaw RJ, Paez JG, Curto M, et al. The Nf2 tumor suppressor, merlin, functions in Rac-dependent signaling. Dev Cell. 2001; 1:63-72. 
13. Scoles DR. The merlin interacting proteins reveal multiple targets for NF2 therapy. Biochim Biophys Acta. 2008;1785:32-54.

14. Cooper J, Li W, You L, et al. Merlin/NF2 functions upstream of the nuclear E3 ubiquitin ligase CRL4DCAF1 to suppress oncogenic gene expression. Sci Signal. 2011;4:pt6.

15. Curto M, McClatchey AI. Nf2/Merlin: a coordinator of receptor signalling and intercellular contact. Br J Cancer. 2008;98:256-62.

16. Maitra S, Kulikauskas RM, Gavilan H, Fehon RG. The tumor suppressors Merlin and expanded function cooperatively to modulate receptor endocytosis and signaling. Curr Biol. 2006;16:702-9.

17. Curto M, Cole BK, Lallemand D, Liu CH, McClatchey AI. Contact-dependent inhibition of EGFR signaling by Nf2/Merlin. J Cell Biol. 2007;177:893-903.

18. James MF, Beauchamp RL, Manchanda N, Kazlauskas A, Ramesh V. A NHERF binding site links the betaPDGFR to the cytoskeleton and regulates cell spreading and migration. J Cell Sci. 2004;117:2951-61.

19. McClatchey AI, Fehon RG. Merlin and the ERM proteins-regulators of receptor distribution and signaling at the cell cortex. Trends Cell Biol. 2009;19:198-206.

20. Holbro T, Hynes NE. ErbB receptors: directing key signaling networks throughout life. Annu Rev Pharmacol Toxicol. 2004;44:195-217.

21. Hansen MR, Linthicum FH Jr. Expression of neuregulin and activation of erbB receptors in vestibular schwannomas: possible autocrine loop stimulation. Otol Neurotol. 2004;25:155-9.

22. Lallemand D, Manent J, Couvelard A, et al. Merlin regulates transmembrane receptor accumulation and signaling at the plasma membrane in primary mouse Schwann cells and in human schwannomas. Oncogene. 2009;28:854-65.

23. Clark JJ, Provenzano M, Diggelmann HR, Xu N, Hansen SS, Hansen MR. The ErbB inhibitors trastuzumab and erlotinib inhibit growth of vestibular schwannoma xenografts in nude mice: a preliminary study. Otol Neurotol. 2008;29:846-53.

24. Plotkin SR, Halpin C, McKenna MJ, Loeffler JS, Batchelor TT, Barker FG 2nd. Erlotinib for progressive vestibular schwannoma in neurofibromatosis 2 patients. Otol Neurotol. 2010;31:1135-43.

25. Karajannis MA, Legault G, Hagiwara M, et al. Phase II trial of lapatinib in adult and pediatric patients with neurofibromatosis type 2 and progressive vestibular schwannomas. Neuro Oncol. 2012;14:1163-70.

26. Dvorak HF, Brown LF, Detmar M, Dvorak AM. Vascular permeability factor/vascular endothelial growth factor, microvascular hyperpermeability, and angiogenesis. Am J Pathol. 1995; 146:1029-39.

27. Sondell M, Lundborg G, Kanje M. Vascular endothelial growth factor has neurotrophic activity and stimulates axonal outgrowth, enhancing cell survival and Schwann cell proliferation in the peripheral nervous system. J Neurosci. 1999;19:5731-40.

28. Folkman J. Angiogenesis in cancer, vascular, rheumatoid and other disease. Nat Med. 1995;1:27-31.

29. Ferrara N, Keyt B. Vascular endothelial growth factor: basic biology and clinical implications. Exs. 1997;79:209-32.

30. Gerber HP, Dixit V, Ferrara N. Vascular endothelial growth factor induces expression of the antiapoptotic proteins Bcl-2 and A1 in vascular endothelial cells. J Biol Chem. 1998;273:13313-6.

31. Caye-Thomasen P, Baandrup L, Jacobsen GK, Thomsen J, Stangerup SE. Immunohistochemical demonstration of vascular endothelial growth factor in vestibular schwannomas correlates to tumor growth rate. Laryngoscope. 2003;113:2129-34.

32. Komotar RJ, Starke RM, Sisti MB, Connolly ES. The role of bevacizumab in hearing preservation and tumor volume control in patients with vestibular schwannomas. Neurosurgery. 2009; 65:N12.
33. Mautner VF, Nguyen R, Kutta $\mathrm{H}$, et al. Bevacizumab induces regression of vestibular schwannomas in patients with neurofibromatosis type 2. Neuro Oncol. 2010;12:14-8.

34. Plotkin SR, Stemmer-Rachamimov AO, Barker FG 2nd, et al. Hearing improvement after bevacizumab in patients with neurofibromatosis type 2. N Engl J Med. 2009;361:358-67.

35. Wong HK, Lahdenranta J, Kamoun WS, et al. Anti-vascular endothelial growth factor therapies as a novel therapeutic approach to treating neurofibromatosis-related tumors. Cancer Res. 2010;70:3483-93.

36. Mautner VF, Nguyen R, Knecht R, Bokemeyer C. Radiographic regression of vestibular schwannomas induced by bevacizumab treatment: sustain under continuous drug application and rebound after drug discontinuation. Ann Oncol. 2010;21:2294-5.

37. O'Reilly MS, Boehm T, Shing Y, et al. Endostatin: an endogenous inhibitor of angiogenesis and tumor growth. Cell. 1997;88:277-85.

38. Lopez-Lago MA, Okada T, Murillo MM, Socci N, Giancotti FG. Loss of the tumor suppressor gene NF2, encoding merlin, constitutively activates integrin-dependent mTORC1 signaling. Mol Cell Biol. 2009;29:4235-49.

39. James MF, Han S, Polizzano C, et al. NF2/merlin is a novel negative regulator of mTOR complex 1 , and activation of mTORC1 is associated with meningioma and schwannoma growth. Mol Cell Biol. 2009;29:4250-61.

40. Sarbassov DD, Guertin DA, Ali SM, Sabatini DM. Phosphorylation and regulation of $\mathrm{Akt} / \mathrm{PKB}$ by the rictor-mTOR complex. Science. 2005;307:1098-101.

41. Karajannis MA, Legault G, Hagiwara M, et al. Phase II study of everolimus in children and adults with neurofibromatosis type 2 and progressive vestibular schwannomas. Neuro Oncol. 2014;16:292-7.

42. Blakeley JO, Evans DG, Adler J, et al. Consensus recommendations for current treatments and accelerating clinical trials for patients with neurofibromatosis type 2 . Am J Med Genet A. 2012;158A:24-41.

43. Altomare DA, Testa JR. Perturbations of the AKT signaling pathway in human cancer. Oncogene. 2005;24:7455-64.

44. Coffer PJ, Jin J, Woodgett JR. Protein kinase B (c-Akt): a multifunctional mediator of phosphatidylinositol 3-kinase activation. Biochem J. 1998;335(Pt 1):1-13.

45. Toker A, Newton AC. Cellular signaling: pivoting around PDK1. Cell. 2000;103:185-8.

46. Mora A, Komander D, van Aalten DM, Alessi DR. PDK1, the master regulator of AGC kinase signal transduction. Semin Cell Dev Biol. 2004;15:161-70.

47. Yang J, Cron P, Good VM, Thompson V, Hemmings BA, Barford D. Crystal structure of an activated Akt/protein kinase B ternary complex with GSK3-peptide and AMP-PNP. Nat Struct Biol. 2002;9:940-4.

48. Jacob A, Lee TX, Neff BA, Miller S, Welling B, Chang LS. Phosphatidylinositol 3-kinase/AKT pathway activation in human vestibular schwannoma. Otol Neurotol. 2008;29:58-68.

49. Hilton DA, Ristic N, Hanemann CO. Activation of ERK, AKT and JNK signalling pathways in human schwannomas in situ. Histopathology. 2009;55:744-9.

50. Lee TX, Packer MD, Huang J, et al. Growth inhibitory and antitumour activities of OSU-03012, a novel PDK-1 inhibitor, on vestibular schwannoma and malignant schwannoma cells. Eur J Cancer. 2009;45:1709-20.

51. Weng SC, Kashida Y, Kulp SK, et al. Sensitizing estrogen receptor-negative breast cancer cells to tamoxifen with OSU03012, a novel celecoxib-derived phosphoinositide-dependent protein kinase-1/Akt signaling inhibitor. Mol Cancer Ther. 2008;7:800-8. 
52. Wang YC, Kulp SK, Wang D, et al. Targeting endoplasmic reticulum stress and Akt with OSU-03012 and gefitinib or erlotinib to overcome resistance to epidermal growth factor receptor inhibitors. Cancer Res. 2008;68:2820-30.

53. Porchia LM, Guerra M, Wang YC, et al. 2-amino-N-\{4-[5-(2phenanthrenyl)-3-(trifluoromethyl)-1H-pyrazol-1-yl]-phe nyl acetamide (OSU-03012), a celecoxib derivative, directly targets p21-activated kinase. Mol Pharmacol. 2007;72:1124-31.

54. Alvarez FJ, Murahari S, Couto CG, et al. 3-Phosphoinositidedependent protein kinase-1/Akt signalling and inhibition in a canine prostate carcinoma cell line. Vet Comp Oncol. 2007;5:47-58.

55. Zhu J, Huang JW, Tseng PH, et al. From the cyclooxygenase-2 inhibitor celecoxib to a novel class of 3-phosphoinositidedependent protein kinase-1 inhibitors. Cancer Res. 2004; 64:4309-18.

56. Chen CS, Weng SC, Tseng PH, Lin HP. Histone acetylationindependent effect of histone deacetylase inhibitors on Akt through the reshuffling of protein phosphatase 1 complexes. J Biol Chem. 2005;280:38879-87.

57. Lee TX, Packer MD, Huang J, et al. Growth inhibitory and antitumour activities of OSU-03012, a novel PDK-1 inhibitor, on vestibular schwannoma and malignant schwannoma cells. Eur J Cancer. 2009;45:1709-20.

58. Bush ML, Oblinger J, Brendel V, et al. AR42, a novel histone deacetylase inhibitor, as a potential therapy for vestibular schwannomas and meningiomas. Neuro Oncol. 2011;13:983-99.

59. Jacob A, Oblinger J, Bush ML, et al. Preclinical validation of AR42, a novel histone deacetylase inhibitor, as treatment for vestibular schwannomas. Laryngoscope. 2012;122:174-89.

60. Vallières GB, Burroughs P. Aeterna Zentaris to discontinue phase 3 trial in multiple myeloma with perifosine following data safety monitoring board recommendation. Available at: http://www. aezsinc.com/en/page.php? $\mathrm{p}=60 \& \mathrm{q}=550$. Accessed April 26, 2014.

61. Miller C, Igarashi S, Jacob A. Molecular pathogenesis of vestibular schwannomas: insights for the development of novel medical therapies. Otolaryngol Polska. 2012;66:84-95.

62. Sells MA, Chernoff J. Emerging from the Pak: the p21-activated protein kinase family. Trends Cell Biol. 1997;7:162-7.

63. Xiao GH, Beeser A, Chernoff J, Testa JR. p21-activated kinase links Rac/Cdc42 signaling to merlin. J Biol Chem. 2002; 277:883-6.

64. Kissil JL. Merlin phosphorylation by p21-activated kinase 2 and effects of phosphorylation on merlin localization. J Biol Chem. 2002;277:10394-9.

65. Edelman GM. A golden age for adhesion. Cell Adhes Commun. 1993;1:1-7.

66. Tysnes BB, Mahesparan R. Biological mechanisms of glioma invasion and potential therapeutic targets. J Neurooncol. 2001; 53:129-47.

67. Hamel W, Westphal M. Growth factors in gliomas revisited. Acta Neurochir (Wien). 2000;142:113-37.

68. Kaempchen K, Mielke K, Utermark T, Langmesser S, Hanemann CO. Upregulation of the Rac1/JNK signaling pathway in primary human schwannoma cells. Hum Mol Genet. 2003;12:1211-21.

69. Yi C, Wilker EW, Yaffe MB, Stemmer-Rachamimov A, Kissil JL. Validation of the p21-activated kinases as targets for inhibition in neurofibromatosis type 2. Cancer Res. 2008;68:7932-7.

70. Flaiz C, Ammoun S, Biebl A, Hanemann CO. Altered adhesive structures and their relation to RhoGTPase activation in merlindeficient Schwannoma. Brain Pathol. 2009;19:27-38.

71. Kissil JL, Wilker EW, Johnson KC, Eckman MS, Yaffe MB, Jacks T. Merlin, the product of the Nf2 tumor suppressor gene, is an inhibitor of the p21-activated kinase, Pak1. Mol Cell. 2003;12:841-9.
72. Bokoch GM. PAK'n it in: identification of a selective PAK inhibitor. Chem Biol. 2008;15:305-6.

73. Flaiz C, Chernoff J, Ammoun S, Peterson JR, Hanemann CO. PAK kinase regulates Rac GTPase and is a potential target in human schwannomas. Exp Neurol. 2009;218:137-44.

74. Calderwood SK. Molecular cochaperones: tumor growth and cancer treatment. Scientifica. 2013;2013:217513.

75. Whitesell L, Lindquist SL. HSP90 and the chaperoning of cancer. Nat Rev Cancer. 2005;5:761-72.

76. Tanaka K, Eskin A, Chareyre F, et al. Therapeutic potential of HSP90 inhibition for neurofibromatosis type 2. Clin Cancer Res. 2013;19:3856-70.

77. Nobili S, Lippi D, Witort E, et al. Natural compounds for cancer treatment and prevention. Pharmacol Res. 2009;59:365-78.

78. Spear SA, Burns SS, Oblinger JL, et al. Natural compounds as potential treatments of NF2-deficient schwannoma and meningioma: cucurbitacin $\mathrm{D}$ and goyazensolide. Otol Neurotol. 2013;34:1519-27.

79. Petrilli A, Bott M, Fernandez-Valle C. Inhibition of SIRT2 in merlin/NF2-mutant Schwann cells triggers necrosis. Oncotarget. 2013;4:2354-65.

80. Knight D, Tiersten A, Miao H, Llorente M, Elfring G, Novik Y, Speyer J, Volm M, Miller L. PTC299, a novel regulator of Tumor VEGF expression is well tolerated and achieves target plasma concentrations: dose-ranging results of a phase $1 \mathrm{~b}$ study in women with metastatic breast cancer. Cancer Research. 2009;. doi:10.1158/0008-5472.

81. - Shin YK, et al. Grb2-associated binder-1 is required for neuregulin-1-induced peripheral nerve myelination. J Neurosci. 2014;34(22): 7657-62. Grb2-associated binders (Gabs) are scaffolding proteins implicated in cell signaling via receptor tyrosine kinases including neuregulin-1(NRG1)-ErbB receptor signaling, which is essential for peripheral nerve myelination. ErbB receptor signaling is essential for Schwann cell myelination. Knockdown of a vital ErbB scaffolding protein, Grb2associated binders (Gab1), causes hypomyelination in Schwann cells in the peripheral nervous system. The findings suggest that Gabl, and thus ErbB receptor signaling, is essential in peripheral nerve myelin development and structure.

82. - London NR, Gurgel RK. The role of vascular endothelial growth factor and vascular stability in diseases of the ear. Laryngoscope. 2013. The Anti-VEGF agent bevacizumab has demonstrated efficacy against vestibular schwannomas in regard to tumor size reduction and hearing improvement in NF2 patients. Loss of Merlin in NF2 results in a decrease in the expression of the anti-angiogenic protein $S E M A 3 F$, a possible mechanism whereby VEGF inhibitors decrease angiogenesis in NF2-null tumors such as schwannomas.

83. • Nunes FP, et al. Bevacizumab treatment for meningiomas in NF2: a retrospective analysis of 15 patients. PLoS One. 2013;8(3): e59941. In NF2 patients, use of the anti-VEGF agent Bevacizumab can result in tumor shrinkage of progressive vestibular schwannomas. This study suggested that tumor size reduction was a temporary effect with the median duration of response of 3.7 months and median time to progression of 15 months. Authors found that meningiomas in studied NF2 patients were not affected by bevacizumab treatment.

84. - James MF, et al. Regulation of mTOR complex 2 signaling in neurofibromatosis 2-deficient target cell types. Mol Cancer Res. 2012;10(5): 649-59. Merlin positively regulates the kinase activity of mTORC2 and downstream phosphorylation of mTORC2 substrates, namely AKT, is reduced in merlin-deficient cells. Two specific inhibitors of mTOR were examined to help identify the most efficacious class of compounds for inhibition of mTOR mediated signaling and proliferation in NF2 deficient cell lines. 
85. • Jacob A, et al. Preclinical validation of AR42, a novel histone deacetylase inhibitor, as treatment for vestibular schwannomas. Laryngoscope. 2012;122(1): 174-89. Vestibular schwannomas rely on PI3 K/AKT activation to promote cell proliferation and survival. AR42, a novel histone deacetylase inhibitor that suppresses AKT signaling, suppressed schwannoma growth at doses correlating with AKT dephosphorylation, cell cycle arrest and apoptosis.
86. - Menges CW, et al. Group I p21-activated kinases (PAKs) promote tumor cell proliferation and survival through the AKT1 and Raf-MAPK pathways. Mol Cancer Res. 2012;10(9): 1178-88. p21-activated kinases (PAKs) are important regulators of cell motility, survival, proliferation, and gene transcription. Group I PAKs are activated in many cancer cell lines. Inhibition of PAK results in attenuation of AKT and Raf-MAPK signaling and decreased tumor cell viability. 